\title{
Calculation of DWPF Canister Decay Heat for Sludge Macro-Batches 1B to 9
}

by

A. S. Choi

Westinghouse Savannah River Company

Savannah River Site

Aiken, South Carolina 29808

This paper was prepared in connection with work done under the above contract number with the U.S. Department of Energy. By acceptance of this paper, the publisher and/or recipient acknowledges the U.S. Government's right to retain a nonexclusive, royalty-free license in and to any copyright covering this paper, along with the right to reproduce and to authorize others to reproduce all or part of the copyrighted paper. 


\section{DISCLAIMER}

This report was prepared as an account of work sponsored by an agency of the United States Government. Neither the United States Government nor any agency thereof, nor any of their employees, makes any warranty, express or implied, or assumes any legal liability or responsibility for the accuracy, completeness, or usefulness of any information, apparatus, product, or process disclosed, or represents that its use would not infringe privately owned rights. Reference herein to any specific commercial product, process, or service by trade name, trademark, manufacturer, or otherwise does not necessarily constitute or imply its endorsement, recommendation, or favoring by the United States Government or any agency thereof. The views and opinions of authors expressed herein do not necessarily state or reflect those of the United States Government or any agency thereof.

This report has been reproduced directly from the best available copy.

Available to DOE and DOE contractors from the Office of Scientific and Technical Information, P.O. Box 62, Oak Ridge, TN 37831; prices available from (615) 576-8401.

Available to the public from the National Technical Information Service, U.S. Department of Commerce, 5285 Port Royal Road, Springfield, VA 22161. 


\section{DISCLAIMER}

Portions of this document may be illegible in electronic image products. Images are produced from the best available original document. 
WSRC-TR-98-00466

Keywords: DWPF, Canister, Decay

Heat, HLW System Plan

Revision 9, Macro-Batches

Retention Period: Permanent

\section{CALCULATION OF \\ DWPF CANISTER DECAY HEAT FOR}

SLUDGE MACRO-BATCHES 1B TO 9 (U)

February 18, 1999

Alexander S. Choi

Immobilization Technology Section

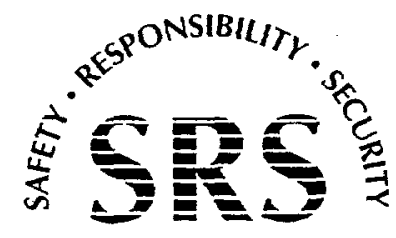


This page left intentionally blank. 


\section{SUMMARY}

The rates of heat generation of DWPF glass canisters due to radioactive decay have been estimated for the remaining nine macro-batches of sludge feed. The estimated decay heat ranged from 177.5 Watts for each Macro-batch 1B canister to 498.4 Watts for each Macro-batch 8 canister. These projections are based on the HLW radionuclide inventory data available as of $4 / 1 / 98$, and do not reflect further decay since then. It was assumed that each DWPF glass canister would also contain a nominal quantity of salt waste based on the reference coupled feed flowsheet. Issue of this report successfully closes a recent technical assistance request (HLW/DWPF-TAR-990003).

\section{INTRODUCTION}

DWPF successfully completed the first macro-batch campaign (1A) in October 1998, and produced a total of 492 canisters, each containing about $3,810 \mathrm{lb}$ of glass on the average; ${ }^{1}$ on a $4,000 \mathrm{lb}$ of glass-per-canister basis, this is equivalent to 469 canisters. This adjusted actual canister yield for Macro-batch $1 \mathrm{~A}$ was very close to the theoretical prediction of 466 made by the CPES model. ${ }^{2}$ Currently, DWPF is processing the second macro-batch (1B) at a rate of about 250 canisters per year. Once sealed and decontaminated, these canisters must be stored on-site temporarily, until they are shipped to a federal repository for permanent disposal. In anticipation of potentially a long delay in the opening of such a repository, DWPF is now designing a second Glass Waste Storage Building (GWSBII). It was the objective of this study to provide the radioactive decay heat projections for all future canisters in support of GWSB-II design.

The baseline glass compositions used in this study to estimate the decay heat were those calculated earlier using the CPES model in support of the HLW System Plan Revision 9. ${ }^{3}$ The baseline glass compositions were calculated based on the sludge processing schedule outlined in the HLW System Plan, which calls for processing the entire sludge inventory in ten macro-batches. ${ }^{2}$ Table 1 shows the makeup of each macro-batch and the projected canister yields.

The chemical composition of each macro-batch thus defined was developed from the tank-by-tank waste characterization system (WCSystem) database available as of October $1997,{ }^{4}$ and was used in the CPES model to calculate a complete HLW flowsheet material balance for each macro-batch, including the baseline glass compositions. ${ }^{3}$ The resulting material balance predicted the total canister yield of 5,084 for the entire duration of sludge campaign, each canister containing $4,000 \mathrm{lb}$ of glass. ${ }^{2}$ Glass blending was optimized in each CPES run using the Product Composition Control System (PCCS), ${ }^{5}$ so the total canister yield predicted by the model represents the theoretical minimum required to convert the entire sludge inventory into glass at the highest waste loading.

During this study, two major modifications were made to the calculated baseline glass compositions. First, the CPES model was run based on the assumption that all macrobatches of sludge would be fed to DWPF along with the tetraphenyl borate (TPB) precipitate produced from the average salt feed for the first 10 years of planned in-tank precipitation (ITP) operation. This hypothetical salt feed is called the 10-year average 
salt; its composition was developed in the 1980's, and has been used in several design basis material balances for DWPF. ${ }^{6}$ Recently, however, the composition of a new blend salt was developed using the WCSystem database available as of April 1998 in support of the salt disposition alternatives down-selection process, ${ }^{7}$ and its impact on the total canister yield was also assessed. ${ }^{8}$

The composition of the new blend salt thus developed is compared in Table A-1 against to that of the 10-year average (called the old blend salt in this memo). For the purpose of this study, the important species to follow are soluble $\mathrm{Cs}$ and $\mathrm{Sr}$ and insoluble solids containing $\mathrm{Pu}$ and $\mathrm{Sr}$. The soluble and insoluble species shown in Table A-1 are further combined into the elemental and ionic distributions in Table A-2; it can be clearly seen that the concentrations of all actinides and most fission products except Cs are significantly higher in the new blend salt than in the old. As a result, it became necessary in this study to revise the salt (or PHA) fraction of the baseline glass compositions using the mass ratios given in Table 3 for an accurate projection of radioactive decay heat.

Second, the concentration of Cs given in the WCSystem database available as of October 1997 were found to be erroneously high for the supernate phase of each tank, thus impacting the soluble $\mathrm{Cs}$ level in each macro-batch and eventually the baseline glass compositions. As a result, a volume-averaged reduction factor of $\mathrm{Cs}$ was calculated in this study for each macro-batch using the WCSystem database available as of April 1998 and applied to the sludge fraction of $\mathrm{Cs}$ in each glass matrix.

TABLE 1. Sludge Processing Schedule Used in HLW System Plan Revision 9.

\begin{tabular}{llr}
\hline Macro-Batch & Tanks & $\begin{array}{r}\text { Canister Yield } \\
\text { @ } 000 \text { b glass/can }\end{array}$ \\
\hline 1A & 51 & $467^{\sharp}$ \\
1B & 42 & $572^{*}$ \\
2 & 8,40 & 479 \\
3 & $7(70 \%), 11,18,19$ & 591 \\
4 & $4,7(30 \%), 12,14$ & 527 \\
5 & $5,6,13(30 \%), 15,21,22$ & 497 \\
6 & $13(70 \%), 23,47$ & 740 \\
7 & $26,32,33$ & 473 \\
8 & $34,35,39,43,40$ heel & 467 \\
9 & $1,2,3,9,10,36,41,42$ heel, 51 heel & 416 \\
\hline
\end{tabular}

* Actual DWPF data after adjustment to 4,000 lb glass/canister.

* Data taken from Ref. 1 and adjusted to 4,000 lb glass/canister. 


\section{BASES}

The WCSystem database contains the inventory information for 41 radionuclide isotopes, and the CPES model tracks only the major radionuclides involving $\mathrm{Cs}, \mathrm{Sr}, \mathrm{Pu}, \mathrm{Tc}$ and $\mathrm{U}$. For most sludge tanks, the Sr-90/Y-90 pair alone accounts for close to $90 \%$ or more of the total radioactivity in a tank. Excluding Tank 51, however, there are eight or so tanks in which the Sr-90/Y-90 pair accounts for less than $80 \%$ of the total radioactivity. In order to identify additional nuclides that contribute significantly to the total radioactivity in those tanks, Table A-3 was constructed from the April 1998 WCSystem database by adding different radionuclides to the $\mathrm{Sr}-90 / \mathrm{Y}-90$ pair. As shown by the last column in Table A-3, nearly all radioactivity in every tank except Tank 19 is accounted for when Cs-137/Ba-137m, Pm-147, Pu-238, Am-241 and Cm-244 are included in addition to Sr$90 / Y-90$. For Tank 19, it is shown that only $90 \%$ of the total radioactivity is accounted for by these radionuclides, since Pu-241 which accounts for nearly $9 \%$ of the total radioactivity in the tank was not included. However, Pu-241 has more than two orders of magnitude lower radioisotopic power than Pu-238, so its omission from the decay heat calculations is justified.

As a result, the following radionuclides were selected for all decay heat calculations made in this study:

$$
\begin{aligned}
& \text { Sr-90/Y-90 } \\
& \text { Cs-137/Ba-137m }
\end{aligned}
$$

Pm-147

$\mathrm{Pu}-238$

Am-241

$\mathrm{Cm}-244$

Calculations showed that as of April 1998 these radionuclides contributed to more than $96 \%$ of the total decay heat generated in all tanks. Additional key bases used in the calculations include:

- All radioactive decay modes are frozen as of April 1998.

- Each canister produced contains $4,000 \mathrm{lb}$ of glass consisting of both sludge and salt oxides according to the reference coupled feed flowsheet. ${ }^{6}$ The fraction of salt oxides in glass is nominally $6.1 \mathrm{wt} \%{ }^{3}$

- The chemical composition of the salt feed to the ITP is that of the old blend salt.

- The concentrations of $\mathrm{Cs}, \mathrm{Pu}$ and $\mathrm{Sr}$ in the salt fraction of each baseline glass matrix were multiplied by the following ratios found in Table A-2:

$\begin{array}{lr}\mathrm{Cs} & 0.91 \\ \mathrm{Pu} & 16.01 \\ \mathrm{Sr} & 31.50\end{array}$


- The following wt\% distributions of Sr-90 and Cs-137 were assumed in both sludge and salt feeds:

$$
\begin{array}{ll}
\text { Sr-90 } & 36.86 w t \% \text { of total } \mathrm{Sr} \\
\mathrm{Cs}-137 & 22.56 \mathrm{wt} \% \text { of total Cs }
\end{array}
$$

- The following distribution of $\mathrm{Pu}$ isotopes in each macro-batch glass matrix was obtained by mass-averaging contributions from all constituent tanks (see Sample Calculations in Appendix):

TABLE 2. Calculated Mass-Averaged Pu Isotope Distributions (wt\% total Pu).

\begin{tabular}{lll}
\hline Macro-Batch & Pu-238 & Pu-239 \\
\hline 1B & 5.96 & 85.64 \\
2 & 0.22 & 95.29 \\
3 & 9.38 & 81.23 \\
4 & 8.62 & 81.33 \\
5 & 7.27 & 82.49 \\
6 & 2.98 & 89.79 \\
7 & 14.34 & 74.0 \\
8 & 21.79 & 63.86 \\
9 & 11.37 & 78.05 \\
\hline
\end{tabular}

- The concentration of $\mathrm{Cs}$ in the sludge fraction of each macro-batch glass matrix was multiplied by the following volume-averaged reduction factors to account for the error found in the October 1997 WCSystem supernate database:

TABLE 3. Volume-Averaged Reduction Factors for Soluble Cs in Sludge Feed.

\begin{tabular}{ll}
\hline Macro-Batch & Reduction Factor \\
\hline 1 B & N/A \\
2 & 0.00267 \\
3 & 0.00444 \\
4 & 0.01938 \\
5 & 0.00188 \\
6 & 0.00579 \\
7 & 0.00164 \\
8 & 0.00311 \\
9 & 0.00814 \\
\hline
\end{tabular}

** As a comparison, DWPF isotopic data issued in 1985 showed the following wt\% distributions: ${ }^{9}$

$\begin{array}{lcc} & \text { sludge } & \text { salt } \\ \text { Sr-90 } & 59.5 & 53.5 \\ \text { Cs-137 } & 44.6 & 39.0\end{array}$


- The concentrations of Pm-147, Am-241 and Cm-244 in glass were estimated by multiplying the composite mass ratio of each nuclide to Pu-239 in each macro-batch with the mass of Pu-239 in the sludge fraction of glass (see Sample Calculations in Appendix).

- The modified concentrations of those selected radionuclides in the baseline glass matrices were multiplied by the following radioisotopic power data to calculate the decay heat: ${ }^{10}$

\section{TABLE 4. Radioisotopic Power Data Used in Decay Heat Calculations.}

\begin{tabular}{|c|c|}
\hline Nuclide & Watt/gm \\
\hline $\mathrm{Sr}-90^{+}$ & 0.916 \\
\hline $\mathrm{Cs}-137^{+}$ & 0.427 \\
\hline Pm-147 & 0.340 \\
\hline Pu-238 & 0.558 \\
\hline Am-241 & 0.113 \\
\hline $\mathrm{Cm}-244$ & 2.78 \\
\hline
\end{tabular}

\section{RESULTS}

The radioactive decay heat projections calculated using the bases discussed above are shown in Table 5. These projections include both primary and gamma radiations emitted as of April 1998 by those nuclides selected above plus all of their daughters. It is shown that the projected decay heat ranges from 177.5 Watts for each Macro-batch 1B canister that DWPF is currently producing to 498.4 Watts for each Macro-batch 8 canister. As a comparison, the decay heat calculated earlier for the blend sludge-precipitate glass was about 500 Watts per canister for a 10-year old sludge; ${ }^{9}$ although not mentioned in the reference, it is believed that each canister was assumed to contain $3,700 \mathrm{lb}$ of glass.

Table 5 also shows that the last three macro-batches together generate nearly $50 \%$ of all decay heat, and the contributions by sludge and salt components to the total decay heat are quite comparable except for these three macro-batches along with Macro-batches 1B. The decay heat projections are further broken down into contributions by individual sludge and salt components in Tables 6 and 7. As expected, Sr-90 and its daughter radiations are shown to account for $90 \%$ or more of the decay heat generated by all sludge components except for the last three macro-batches.

Table 7 shows that for Macro-batches 8 and 9 the combined contributions by Pm-147, Am-241 and Cm-244 are 13.4 and 18.0\%, respectively, of the total decay heat generated by all sludge components. It is also shown in Table 7 that 30 to $40 \%$ of the total decay heat generated by all salt components are due to $\mathrm{Sr}-90$ and its daughter radiations, while the remaining 60 to $70 \%$ is due to Cs- 137 and its daughter radiations. Pu-238 is shown to contribute significantly to the total decay heat only in Marco-batch 8 . 
TABLE 5. Calculated Radioactive Decay Heat Projections.

\begin{tabular}{lrrr}
\hline Macro-batch & $\begin{array}{r}\text { Decay Heat } \\
\text { (Watts/Canister) }\end{array}$ & $\begin{array}{r}\text { Salt Contribution } \\
\text { (\% total Watts) }\end{array}$ & $\begin{array}{r}\text { Sludge Contribution } \\
\text { (\% total Watts) }\end{array}$ \\
\hline 1B & 177.5 & 79.6 & 20.4 \\
2 & 294.4 & 50.2 & 49.8 \\
3 & 307.5 & 51.5 & 48.5 \\
4 & 331.9 & 48.7 & 51.3 \\
5 & 319.5 & 47.7 & 52.3 \\
6 & 249.2 & 56.5 & 43.5 \\
7 & 417.0 & 41.3 & 58.7 \\
8 & 498.4 & 36.2 & 63.8 \\
9 & 434.5 & 40.3 & 59.7 \\
\hline
\end{tabular}

TABLE 6. Contributions to Total Decay Heat by Individual Radionuclides (\% total Watts).

\begin{tabular}{|c|c|c|c|c|c|c|c|c|c|}
\hline \multirow[b]{2}{*}{ Macro-batch } & \multicolumn{4}{|c|}{ salt components } & \multicolumn{5}{|c|}{ sludge components } \\
\hline & $\mathrm{Cs}^{+}$ & $\mathrm{Pu}$ & $\mathrm{Sr}^{+}$ & total & $\mathrm{Cs}^{+}$ & $\mathrm{Pm} / \mathrm{Am} / \mathrm{Cm}$ & $\mathrm{Pu}$ & $\mathrm{Sr}^{+}$ & total \\
\hline 1B & 55.0 & 0.5 & 24.0 & 79.6 & 0.1 & 0.9 & 1.3 & 18.2 & 20.4 \\
\hline 2 & 29.7 & 0.0 & 20.4 & 50.2 & 0.1 & 0.4 & 0.0 & 49.4 & 49.8 \\
\hline 3 & 31.8 & 0.7 & 19.0 & 51.5 & 0.1 & 1.4 & 2.3 & 44.8 & 48.5 \\
\hline 4 & 29.4 & 0.6 & 18.6 & 48.7 & 0.2 & 1.3 & 2.2 & 47.6 & 51.3 \\
\hline 5 & 30.6 & 0.4 & 16.8 & 47.7 & 0.0 & 2.9 & 1.3 & 48.0 & 52.3 \\
\hline 6 & 39.2 & 0.2 & 17.1 & 56.5 & 0.0 & 0.6 & 0.6 & 42.2 & 43.5 \\
\hline 7 & 23.4 & 1.2 & 16.7 & 41.3 & 0.0 & 3.8 & 5.6 & 49.3 & 58.7 \\
\hline 8 & 19.6 & 2.7 & 13.9 & 36.2 & 0.0 & 8.5 & 13.7 & 41.6 & 63.8 \\
\hline 9 & 22.5 & 0.9 & 16.9 & 40.3 & 0.7 & 10.8 & 3.9 & 44.3 & 59.7 \\
\hline
\end{tabular}

+ Includes daughter radiations.

TABLE 7. Contributions by Individual Radionuclides to Total Sludge or Salt Decay Heat (\% total Watts by Sludge or Salt).

\begin{tabular}{|c|c|c|c|c|c|c|c|c|c|}
\hline \multirow[b]{2}{*}{ Macro-batch } & \multicolumn{4}{|c|}{ salt components } & \multicolumn{5}{|c|}{ sludge components } \\
\hline & $\mathrm{Cs}^{+}$ & $\mathrm{Pu}$ & $\mathrm{Sr}^{+}$ & total & $\mathrm{Cs}^{+}$ & $\mathrm{Pm} / \mathrm{Am} / \mathrm{Cm}$ & $\mathrm{Pu}$ & $\mathrm{Sr}^{+}$ & total \\
\hline 1B & 69.1 & 0.7 & 30.2 & 100.0 & 0.5 & 4.2 & 6.2 & 89.1 & 100.0 \\
\hline 2 & 59.2 & 0.0 & 40.7 & 100.0 & 0.2 & 0.7 & 0.1 & 99.0 & 100.0 \\
\hline 3 & 61.7 & 1.3 & 37.0 & 100.0 & 0.1 & 2.9 & 4.7 & 92.3 & 100.0 \\
\hline 4 & 60.5 & 1.2 & 38.3 & 100.0 & 0.3 & 2.6 & 4.3 & 92.8 & 100.0 \\
\hline 5 & 64.0 & 0.7 & 35.2 & 100.0 & 0.1 & 5.6 & 2.5 & 91.9 & 100.0 \\
\hline 6 & 69.4 & 0.3 & 30.3 & 100.0 & 0.1 & 1.4 & 1.4 & 97.1 & 100.0 \\
\hline 7 & 56.7 & 3.0 & 40.3 & 100.0 & 0.0 & 6.4 & 9.6 & 84.0 & 100.0 \\
\hline 8 & 54.2 & 7.5 & 38.3 & 100.0 & 0.1 & 13.4 & 21.4 & 65.1 & 100.0 \\
\hline 9 & 55.8 & 2.3 & 42.0 & 100.0 & 1.1 & 18.0 & 6.6 & 74.3 & 100.0 \\
\hline
\end{tabular}

+ Includes daughter radiations. 


\section{CONCLUSION}

The decay heat projections given in Table 5 are valid as of April 1998, and further decay since then was not accounted for. Furthermore, calculations showed that those eight radionuclides chosen for this study accounted for over $96 \%$ of the total decay heat generated in all waste tanks; the remaining $4 \%$ is mainly due to the decay of Co-60 and Eu-154. Therefore, it can be concluded that the design of GWSB-II based on the decay. heat projections given for the last three macro-batches would be conservative. 


\section{REFERENCES}

1. Occhipinti, J. E., Shah, H. B., Lee, E. D., and Choi, A. S., "Preliminary Evaluation of DWPF Canisters Predicted vs DWPF Canisters Poured," HLW-DEN-99-0074, March 4, 1999.

2. Davis, N. E., and Wells, M. N., "High Level Waste System Plan Revision 9 (U)," HLW-OVP-98-0037, April 1998.

3. Choi, A. S., Unpublished Results of CPES Runs, March 1998.

4. Hester, J. R., "High Level Waste Characterization System (WCS)," WSRC-TR-96-0264 (Revision 0), December 1996.

5. Brown, K. G. and Postles, R. L., "SME Acceptability Determination for DWPF Process Control," WSRC-TR-95-0364 (Revision 3), February 21, 1996.

6. Choi, A. S., "Material Balance Tables for the DWPF Coupled Feed Flowsheet with Batch One Sludge (U)," WSRC-TR-93-203 (Rev. 0), August 19, 1993.

7. Rutland, P. L., Fowler, J. R., Beck, S., Choi, A. S., Jacobs, R. A., Elder, H. H., Taylor, G. A., and Duarte, O. E., "Bases, Assumptions, and Results of the Flowsheet Calculations for the Initial Eighteen Salt Disposition Alternatives," WSRC-RP-98-00166 (Revision 0), June 1998.

8. Choi, A. S., "Impact of New Blend Salt Feed Composition on Canister Yield," SRT-PCC-98-0013, August 19, 1998.

9. Fowler, J. R., “DWPF Isotopic Data," DPST-84-971, February 21, 1985.

10. Nuclides and Isotopes, Fourteenth Edition, GE Nuclear Energy, San Jose, California (1989).

11. Accountability Manual Accountability Standard Decay and Activity Values (U), DPSOP 23-11 Revision 5, September 1985. 


\section{APPENDIX}

TABLE A-1. Compositions of New and Old Blend Salt Feeds Calculated by CPES Model.

\begin{tabular}{|c|c|c|c|c|c|c|c|}
\hline insolubles & New (wto dry) & Old (wt\% dry) & Ratio (New/Old) & solubles & New (wt\% dry) & Old (wt\% dry) & Ratio (New/Old) \\
\hline Ag20 & 2.2449E-04 & $3.9622 \mathrm{E}-05$ & 5.67 & $\mathrm{NaNO} 2$ & $9.2300 \mathrm{E}+00$ & $1.2675 \mathrm{E}+01$ & 0.73 \\
\hline $\mathrm{Al} 2 \mathrm{O} 3$ & $2.7638 \mathrm{E}-02$ & $5.3673 \mathrm{E}-03$ & 5.15 & NaNO3 & $4.6978 \mathrm{E}+01$ & $5.0922 \mathrm{E}+01$ & 0.92 \\
\hline $\mathrm{BaSO} 4$ & $5.1104 \mathrm{E}-04$ & $1.0089 \mathrm{E}-04$ & 5.07 & $\mathrm{NaOH}$ & $2.2612 \mathrm{E}+01$ & $1.2543 \mathrm{E}+01$ & 1.80 \\
\hline $\mathrm{Ca} 3(\mathrm{PO} 4) 2$ & $2.5552 \mathrm{E}-04$ & $3.2406 \mathrm{E}-05$ & 7.89 & $\mathrm{Na} 2 \mathrm{CO} 3$ & $4.2202 \mathrm{E}+00$ & $5.3637 E+00$ & 0.79 \\
\hline $\mathrm{CaC} 2 \mathrm{O} 4$ & $1.3440 \mathrm{E}-03$ & $1.5053 \mathrm{E}-04$ & 8.93 & $\mathrm{Na} 2 \mathrm{SO} 4$ & $5.2664 \mathrm{E}+00$ & $6.1158 \mathrm{E}+00$ & 0.86 \\
\hline $\mathrm{CaCO}$ & $4.9058 \mathrm{E}-03$ & $1.6142 \mathrm{E}-03$ & 3.04 & $\mathrm{NaA}(\mathrm{OH}) 4$ & $9.2300 \mathrm{E}+00$ & $1.0390 \mathrm{E}+01$ & 0.89 \\
\hline $\mathrm{CaCO} 3(14)$ & $3.8174 \mathrm{E}-10$ & $5.6242 \mathrm{E}-11$ & 6.79 & $\mathrm{NaCl}$ & $3.9084 \mathrm{E}-01$ & $3.8228 \mathrm{E}-01$ & 1.02 \\
\hline $\mathrm{CaF} 2$ & $2.1982 \mathrm{E}-04$ & $3.9659 \mathrm{E}-05$ & 5.54 & $\mathrm{NaF}$ & $3.4113 \mathrm{E}-01$ & $1.9085 \mathrm{E}-01$ & 1.79 \\
\hline $\mathrm{CaO}$ & & & & $\mathrm{Na} 2 \mathrm{C}_{2} \mathrm{O} 4$ & $6.5984 \mathrm{E}-01$ & $3.3169 \mathrm{E}-01$ & 1.99 \\
\hline $\mathrm{CaSO} 4$ & $1.1689 \mathrm{E}-03$ & $7.7484 \mathrm{E}-05$ & 15.09 & $\mathrm{Ba}(\mathrm{OH}) 2$ & $4.0870 E-06$ & $5.2866 \mathrm{E}-03$ & 0.00 \\
\hline $\mathrm{COO}$ & $1.2966 \mathrm{E}-05$ & $2.4177 \mathrm{E}-06$ & 5.36 & $\mathrm{Ca}(\mathrm{OH})_{2}$ & $1.1764 \mathrm{E}-04$ & 4.3401E-04 & 0.27 \\
\hline $\mathrm{Cr} 203$ & $4.5126 \mathrm{E}-04$ & $9.4800 \mathrm{E}-05$ & 4.76 & $\mathrm{CsOH}$ & $5.8154 \mathrm{E}-03$ & $6.4239 \mathrm{E}-03$ & 0.91 \\
\hline $\mathrm{Cs} 2 \mathrm{O}$ & $9.5776 \mathrm{E}-06$ & & & Group A & $1.3743 \mathrm{E}-03$ & 4.5015E-03 & 0.31 \\
\hline $\mathrm{CuO}$ & $1.8291 \mathrm{E}-04$ & $3.3716 \mathrm{E}-05$ & 5.43 & $\mathrm{KNO} 3$ & 7.6803E-02 & $1.5801 \mathrm{E}-04$ & 486.06 \\
\hline $\mathrm{Fe} 2 \mathrm{O} 3$ & $4.2657 \mathrm{E}-02$ & 1.4070E-02 & 3.03 & КOH & $1.7966 \mathrm{E}-01$ & $2.4030 \mathrm{E}-01$ & 0.75 \\
\hline Group B & $2.1800 \mathrm{E}-03$ & $1.1556 \mathrm{E}-05$ & 188.64 & $\mathrm{NH} 4 \mathrm{OH}$ & & & \\
\hline $\mathrm{HgO}$ & 2.3717E-03 & $6.6954 \mathrm{E}-04$ & 3.54 & NH4NO3 & $7.7777 \mathrm{E}-03$ & & \\
\hline $\mathrm{K} 2 \mathrm{O}$ & & & & $\mathrm{Na}(\mathrm{HgO}(\mathrm{OH}))$ & $9.0545 \mathrm{E}-03$ & $1.0291 \mathrm{E}-02$ & 0.88 \\
\hline $\mathrm{La} 2 \mathrm{O} 3$ & $8.9701 \mathrm{E}-04$ & $4.2521 \mathrm{E}-04$ & 2.11 & $\mathrm{Na} 2 \mathrm{~B} 4 \mathrm{O} 7$ & $4.0091 \mathrm{E}-03$ & & \\
\hline $\mathrm{MgO}$ & $3.8564 \mathrm{E}-04$ & $1.3695 \mathrm{E}-04$ & 2.82 & $\mathrm{Na} 2 \mathrm{CO} 3(14)$ & & $5.4517 \mathrm{E}-09$ & \\
\hline $\mathrm{MnO2}$ & $9.2332 \mathrm{E}-03$ & $1.8388 \mathrm{E}-03$ & 5.02 & $\mathrm{Na} 2 \mathrm{CrO4}$ & $1.2209 \mathrm{E}-01$ & $1.6484 \mathrm{E}-0 \mathrm{I}$ & 0.74 \\
\hline $\mathrm{Na2O}$ & $2.6205 \mathrm{E}-0.6$ & & & $\mathrm{Na} 2 \mathrm{M} \infty 04$ & $9.3144 \mathrm{E}-03$ & $2.7171 \mathrm{E}-02$ & 0.34 \\
\hline $\mathrm{NiO}$ & $5.1267 \mathrm{E}-03$ & 2.1025E-04 & 24.38 & $\mathrm{Na} 2 \mathrm{RhO} 4$ & $2.1543 \mathrm{E}-04$ & $6.8569 \mathrm{E}-04$ & 0.31 \\
\hline $\mathrm{PbCO}_{3}$ & $1.3018 \mathrm{E}-04$ & $1.9107 \mathrm{E}-05$ & 6.81 & $\mathrm{Na} 2 \mathrm{RuO} 4$ & $5.3996 \mathrm{E}-03$ & 2.9467E-03 & 1.83 \\
\hline $\mathrm{PbSO}_{4}$ & $3.7297 \mathrm{E}-04$ & $1.6418 \mathrm{E}-04$ & 2.27 & $\mathrm{Na} 2 \mathrm{SiO}_{3}$ & $1.1082 \mathrm{E}-01$ & $1.4385 \mathrm{E}-01$ & 0.77 \\
\hline $\mathrm{PdO}$ & $2.2323 \mathrm{E}-04$ & $3.5708 \mathrm{E}-05$ & 6.25 & $\mathrm{Na} 3 \mathrm{PO} 4$ & $3.2618 \mathrm{E}-01$ & 4.3364E-01 & 0.75 \\
\hline alpha ( $\mathrm{PuO} 2)$ & 8.3365E-05 & $5.5068 \mathrm{E}-06$ & 15.14 & $\mathrm{NaAg}(\mathrm{OH}) 2$ & $1.6611 \mathrm{E}-04$ & $2.3682 \mathrm{E}-0.4$ & 0.70 \\
\hline $\mathrm{RhO} 2$ & & & & $\mathrm{NaI}$ & $3.8564 \mathrm{E}-04$ & $1.0100 \mathrm{E}-04$ & 3.82 \\
\hline $\mathrm{RuO2}$ & $5.3736 \mathrm{E}-04$ & $8.5848 \mathrm{E}-05$ & 6.26 & $\mathrm{NaTcO4}$ & $8.6322 \mathrm{E}-04$ & $2.8132 \mathrm{E}-03$ & 0.31 \\
\hline $\mathrm{SiO} 2$ & $4.4184 \mathrm{E}-03$ & $9.4396 \mathrm{E}-04$ & 4.68 & $\mathrm{Na} 2 \mathrm{PuO} 2(\mathrm{OH}) 4$ & $9.2137 \mathrm{E}-05$ & $5.3343 \mathrm{E}-06$ & 17.27 \\
\hline $\mathrm{SrCO} 3$ & $3.1640 \mathrm{E}-04$ & $2.9797 \mathrm{E}-05$ & 10.62 & $\mathrm{~Pb}(\mathrm{NO}) 2$ & $8.1026 \mathrm{E}-04$ & & \\
\hline $\mathrm{TCO} 2$ & $1.4708 \mathrm{E}-04$ & $1.3273 \mathrm{E}-05$ & 11.08 & $\mathrm{Sr}(\mathrm{OH}) 2$ & $5.3346 \mathrm{E}-04$ & 6.4386E-07 & 828.53 \\
\hline ThO2 & I.8658E-03 & $1.1729 \mathrm{E}-04$ & 15.91 & Tritium* & $2.9162 \mathrm{E}-14$ & $7.3411 \mathrm{E}-10$ & 0.00 \\
\hline $\mathrm{TiO} 2$ & $2.1520 \mathrm{E}-06$ & 8.3904E-06 & 0.26 & $\mathrm{UO} 2(\mathrm{OH}) 2$ & $2.1757 \mathrm{E}-02$ & $3.7568 \mathrm{E}-05$ & 579.15 \\
\hline U3O8 & $1.4295 \mathrm{E}-02$ & $7.9465 \mathrm{E}-04$ & 17.99 & $\mathrm{Y}(\mathrm{OH}) 3^{*}$ & $1.8778 \mathrm{E}-07$ & $6.4900 \mathrm{E}-07$ & 0.29 \\
\hline $\mathrm{Y} 2(\mathrm{CO} 3) 3$ & $3.9116 \mathrm{E}-08$ & $1.8355 \mathrm{E}-09$ & 21.31 & $\mathrm{C} 6 \mathrm{H} 5 \mathrm{OH}$ salt & $3.2128 \mathrm{E}-02$ & & \\
\hline $\mathrm{ZnO}$ & $3.2488 \mathrm{E}-04$ & 9.8028E-05 & 3.31 & C6H5NH2 & $2.1364 \mathrm{E}-04$ & & . \\
\hline $\mathrm{ZrO2}$ & $7.8719 \mathrm{E}-04$ & $20134 \mathrm{E}-04$ & 3.91 & NH3 & & 4.4795E-03 & \\
\hline hyd H2O & $3.2199 \mathrm{E}-02$ & $7.9428 \mathrm{E}-03$ & 4.05 & & & & \\
\hline zeolite & $3.9376 \mathrm{E}-04$ & $1.5354 \mathrm{E}-03$ & 0.26 & & & & \\
\hline \multirow[t]{2}{*}{ tocal insolubles } & $1.5588 \mathrm{E}-01$ & $3.6910 \mathrm{E}-02$ & 4.22 & total solubles & $9.9844 \mathrm{E}+01$ & $9.9963 E+01$ & 1.00 \\
\hline & & & & total solids & $1.0000 E+02$ & $1.0000 \mathrm{E}+02$ & \\
\hline
\end{tabular}


TABLE A-2. Elemental and Anion Compositions of New and Old Blend Salt Feeds.

\begin{tabular}{|c|c|c|c|c|c|c|c|}
\hline elements & old (wt\% dry) & new (wt\% dry) & ratio (new/old) & anions & old (molar) & new (molar) & ratio (new/old) \\
\hline $\mathrm{Ag}$ & $1.918 \mathrm{E}-04$ & $3.198 \mathrm{E}-04$ & 1.67 & NO3 & $2.540 \mathrm{E}+00$ & $2.352 E+00$ & 0.93 \\
\hline $\mathrm{Al}$ & $2.382 E+00$ & $2.128 \mathrm{E}+\infty 0$ & 0.89 & $\mathrm{NO} 2$ & $7.786 \mathrm{E}-01$ & $5.681 \mathrm{E}-01$ & 0.73 \\
\hline $\mathrm{Ba}$ & 4.294E-03 & $3.038 \mathrm{E}-04$ & 0.07 & $\mathrm{OH}$ & $1.719 E+00$ & $2.745 E+00$ & 1.60 \\
\hline $\mathrm{Ca}$ & $1.018 \mathrm{E}-03$ & $3.01 \mathrm{IE}-03$ & 2.96 & $\mathrm{CO} 3$ & $2.144 \mathrm{E}-01$ & $1.692 \mathrm{E}-01$ & 0.79 \\
\hline Co & $1.900 \mathrm{E}-06$ & $1.019 \mathrm{E}-05$ & 5.36 & SO4 & $1.824 \mathrm{E}-01$ & $1.574 \mathrm{E}-01$ & 0.86 \\
\hline $\mathrm{Cr}$ & $5.297 \mathrm{E}-02$ & 3.950E-02 & 0.75 & $\mathrm{C} 204$ & $1.049 \mathrm{E}-02$ & $2.096 \mathrm{E}-02$ & 200 \\
\hline Cs & $5.697 \mathrm{E}-03$ & $5.167 \mathrm{E}-03$ & 0.91 & PO4 & $1.120 \mathrm{E}-02$ & $8.451 \mathrm{E}-03$ & 0.75 \\
\hline Cu & 2.694E-05 & $1.462 \mathrm{E}-04$ & 5.43 & NH4 & $1.115 \mathrm{E}-03$ & $4.121 \mathrm{E}-04$ & 0.37 \\
\hline $\mathrm{Fe}$ & $9.855 \mathrm{E}-03$ & 2.982E-02 & 3.03 & & & & \\
\hline $\mathrm{Hg}$ & $8.667 \mathrm{E}-03$ & $9.277 \mathrm{E}-03$ & 1.07 & & & & \\
\hline 1 & $8.554 \mathrm{E}-05$ & $3.266 \mathrm{E}-04$ & 3.82 & & & & \\
\hline K & $1.675 \mathrm{E}-01$ & $1.549 \mathrm{E}-01$ & 0.92 & & & & \\
\hline La & $3.636 \mathrm{E}-04$ & $9.483 \mathrm{E}-04$ & 2.61 & & & & \\
\hline $\mathrm{Mg}$ & 9.424E-05 & 2.355E-04 & 2.50 & & & & \\
\hline Mn & $1.162 \mathrm{E}-03$ & 5.835E-03 & 5.02 & & & & \\
\hline Mo & $1.641 \mathrm{E}-02$ & $5.487 \mathrm{E}-03$ & 0.33 & & & & \\
\hline $\mathrm{Na}$ & $3.217 \mathrm{E}+01$ & $3.488 \mathrm{E}+01$ & 1.08 & & & & \\
\hline $\mathrm{Ni}$ & $1.652 \mathrm{E}-04$ & $4.030 \mathrm{E}-03$ & 24.39 & & & & \\
\hline$P$ & $8.196 \mathrm{E}-02$ & $6.170 \mathrm{E}-02$ & 0.75 & & & & \\
\hline $\mathrm{Pb}$ & 1.269E-04 & $8.629 \mathrm{E}-04$ & 6.80 & & & & \\
\hline $\mathbf{P d}$ & $3.110 \mathrm{E}-05$ & $1.940 \mathrm{E}-04$ & 6.24 & & & & \\
\hline Pu & 8.190E-06 & $1.311 \mathrm{E}-04$ & 16.01 & & & & \\
\hline Rh & $3.312 \mathrm{E}-04$ & $1.041 \mathrm{E}-04$ & 0.31 & & & & \\
\hline $\mathbf{R u}$ & 1.477E-03 & 2.995E-03 & 2.03 & & & & \\
\hline$s$ & $1.382 E+00$ & $1.191 \mathrm{E}+\infty$ & 0.86 & & & & \\
\hline $\mathrm{Si}$ & 3.385E-02 & $2.763 \mathrm{E}-02$ & 0.82 & & & & \\
\hline Sr & $1.816 \mathrm{E}-05$ & $5.720 \mathrm{E}-04$ & 31.50 & & & & \\
\hline Tc & $1.507 \mathrm{E}-03$ & $5.704 \mathrm{E}-04$ & 0.38 & & & & \\
\hline $\mathrm{Th}$ & $1.031 \mathrm{E}-04$ & $1.640 \mathrm{E}-03$ & 15.91 & & & & \\
\hline $\mathrm{Ti}$ & $5.034 \mathrm{E}-06$ & $1.291 \mathrm{E}-06$ & 0.26 & & & & \\
\hline $\mathrm{U}$ & $7.032 E-04$ & $2.916 \mathrm{E}-02$ & 41.47 & & & & \\
\hline$Y$ & $4.130 \mathrm{E}-07$ & 1.387E-07 & 0.34 & & & & \\
\hline $\mathrm{Zn}$ & 7.871E-05 & $2.609 \mathrm{E}-04$ & 3.31 & & & & \\
\hline $\mathrm{Zr}$ & 1.525E-04 & $1.247 \mathrm{E}-03$ & 8.18 & & & & \\
\hline
\end{tabular}


TABLE A-3. Tank-By-Tank Major Radionuclides Distributions (\% Total Ci)

\begin{tabular}{|c|c|c|c|c|c|c|c|}
\hline Tank & $5 r-90 / Y-90$ & $\begin{array}{c}\mathrm{Sr}-90 / \mathrm{Y}-90 \\
\mathrm{Pu}-238\end{array}$ & $\begin{array}{c}S x-90 / Y-90 \\
P m-147\end{array}$ & $\begin{array}{l}S r-90 / Y-90 \\
A m-241\end{array}$ & $\begin{array}{c}\mathrm{Sr}-90 / \mathrm{Y}-90 \\
\mathrm{Cs}-137 / \mathrm{Ba}-137 \mathrm{~m}\end{array}$ & $\begin{array}{c}\mathrm{Sr}-90 / \mathrm{Y}- \\
90 \\
\mathrm{Pu}-238 \\
\mathrm{Am}-241\end{array}$ & $\begin{array}{c}\mathrm{Sr}-90 / \mathrm{Y}-90 \\
\mathrm{Cs}-137 / \mathrm{Ba}-137 \mathrm{~m} \\
\mathrm{Pm}-147 \mathrm{Pu}-238 \\
\mathrm{Am}-241 \mathrm{Cm}-244\end{array}$ \\
\hline 1 & 93.19083 & 93.19083 & 93.28030 & 93.35120 & 99.54802 & 93.35120 & 99.79790 \\
\hline 2 & 93.24847 & 93.24847 & 93.26543 & 93.42549 & 99.65477 & 93.42549 & 99.84879 \\
\hline 3 & 93.23167 & 93.23167 & 93.26137 & 93.40941 & 99.62005 & 93.40941 & 99.82752 \\
\hline 4 & 91.29367 & 91.29367 & 93.23533 & 91.42257 & 97.43911 & 91.42257 & 99.50970 \\
\hline 5 & 93.08541 & 93.08541 & 93.28432 & 93.23504 & 99.40759 & 93.23504 & 99.75618 \\
\hline 6 & 92.87982 & 92.87982 & 93.28105 & 93.01921 & 99.15643 & 93.01921 & 99.69709 \\
\hline 7 & 93.08884 & 93.08884 & 93.15384 & 93.32400 & 99.45276 & 93.32400 & 99.75295 \\
\hline 8 & 92.35409 & 92.35409 & 93.17583 & 92.52690 & 98.58321 & 92.52690 & 99.57780 \\
\hline 9 & 93.26774 & 93.26774 & 93.28460 & 93.43513 & 99.67557 & 93.43513 & 99.85985 \\
\hline 10 & 93.26440 & 93.26440 & 93.28466 & 93.44310 & 99.63224 & 93.44310 & 99.83124 \\
\hline 11 & 91.92516 & 92.94364 & 92.48549 & 92.41548 & 96.80379 & 93.43396 & 98.87335 \\
\hline 12 & 93.11837 & 93.71321 & 93.31973 & 93.36961 & 98.18884 & 93.96445 & 99.23669 \\
\hline 13 & 93.53910 & 93.77343 & 93.65238 & 93.70182 & 98.98488 & 93.93615 & 99.49549 \\
\hline 14 & 93.44634 & 93.48869 & 93.47929 & 93.60762 & 99.51557 & 93.64997 & 99.75226 \\
\hline 15 & 93.76315 & 94.02995 & 93.91198 & 93.91184 & 98.77745 & 94.17865 & 99.34220 \\
\hline \multicolumn{8}{|l|}{16} \\
\hline 17 & 88.68078 & 88.68078 & 89.70037 & 90.86509 & 94.64921 & 90.86509 & 97.85316 \\
\hline 18 & 88.98413 & 88.98413 & 89.92874 & 91.01609 & 94.98237 & 91.01609 & 97.95897 \\
\hline 19 & 67.36934 & 67.36934 & 68.44054 & 84.67471 & 71.88356 & 84.67471 & 90.26015 \\
\hline \multicolumn{8}{|l|}{20} \\
\hline 21 & 91.39443 & 92.04976 & 93.23713 & 91.44579 & 96.19182 & 92.10112 & 98.74168 \\
\hline 22 & 91.90923 & 92.43779 & 93.41748 & 91.94329 & 96.73451 & 92.47185 & 98.80587 \\
\hline 23 & & & & & 99.88377 & & 99.88377 \\
\hline \multicolumn{8}{|l|}{24} \\
\hline \multicolumn{8}{|l|}{25} \\
\hline 26 & 66.03848 & 66.03848 & 88.82634 & 67.19269 & 70.39276 & 67.19269 & 94.33485 \\
\hline \multicolumn{8}{|l|}{27} \\
\hline \multicolumn{8}{|l|}{28} \\
\hline \multicolumn{8}{|l|}{29} \\
\hline \multicolumn{8}{|l|}{31} \\
\hline 32 & 88.03259 & 89.47497 & 91.29795 & 88.71121 & 92.67007 & 90.15360 & 98.05690 \\
\hline 33 & 71.86873 & 71.86873 & 92.18667 & 71.95267 & 76.60998 & 71.95267 & 97.01191 \\
\hline 34 & 74.40737 & 74.40737 & 92.66879 & 74.49259 & 79.32339 & 74.49259 & 97.67007 \\
\hline 35 & 86.36694 & 87.95937 & 90.74518 & 87.12623 & 90.89603 & 88.71867 & 97.62647 \\
\hline 36 & 88.11661 & 90.40613 & 90.02258 & 89.12357 & 92.74952 & 91.41309 & 97.95245 \\
\hline \multicolumn{8}{|l|}{37} \\
\hline \multicolumn{8}{|l|}{38} \\
\hline 39 & 80.53932 & 82.62128 & 88.96119 & 81.61266 & 84.73911 & 83.69461 & 96.31674 \\
\hline 40 & 91.05650 & 91.42442 & 92.38447 & 91.67955 & 96.24184 & 92.04747 & 98.56113 \\
\hline 41 & 88.82159 & 89.39772 & 93.08065 & 88.85097 & 93.45375 & 89.42710 & 98.31881 \\
\hline 42 & 88.98328 & 90.49288 & 91.22221 & 89.86876 & 94.08944 & 91.37835 & 98.81233 \\
\hline 43 & 78.57810 & 80.43729 & 90.37614 & 78.78849 & 82.65493 & 80.64769 & 96.52303 \\
\hline \multicolumn{8}{|l|}{44} \\
\hline \multicolumn{8}{|l|}{45} \\
\hline \multicolumn{5}{|l|}{46} & 79.95552 & 76.80484 & \\
\hline 41 & 74.99712 & & 80.92513 & & & & \\
\hline \multicolumn{8}{|l|}{$\begin{array}{l}40 \\
49\end{array}$} \\
\hline \multicolumn{8}{|l|}{50} \\
\hline 51 & 59.52562 & 69.04729 & 63.61532 & 62.37863 & 74.78581 & 71.90029 & 97.65535 \\
\hline
\end{tabular}


This page left intentionally blank. 


\section{SAMPLE CALCULATIONS}

All sample calculations are for Marco-batch 4, which consists of Tanks 4, 12, 14, and $30 \%$ of Tank 7 .

Calculation of Isotopic Distribution of Pu-238 and 239

The following data are obtained from the WCSystem database:

\begin{tabular}{|c|rrrrr|}
\hline Tank & Pu-238 (Ci) & Pu-239 (Ci) & Pu-240 (Ci) & Pu-241 (Ci) & Pu-242 (Ci) \\
\hline 4 & & $5.977 \mathrm{E}+02$ & $1.336 \mathrm{E}+02$ & $5.365 \mathrm{E}+02$ & $2.749 \mathrm{E}-02$ \\
7 & & $3.666 \mathrm{E}+03$ & $8.756 \mathrm{E}+02$ & $2.116 \mathrm{E}+03$ & $2.824 \mathrm{E}-01$ \\
12 & $1.203 \mathrm{E}+05$ & $2.316 \mathrm{E}+03$ & $1.280 \mathrm{E}+03$ & $1.532 \mathrm{E}+04$ & $1.849 \mathrm{E}+00$ \\
14 & $2.001 \mathrm{E}+02$ & $6.045 \mathrm{E}+01$ & $1.952 \mathrm{E}+01$ & $2.776 \mathrm{E}+01$ & $3.574 \mathrm{E}-03$ \\
\hline
\end{tabular}

In addition, the following specific curie data in Ci/gm are used: ${ }^{11}$

\begin{tabular}{lllll}
\hline Pu-238 & Pu-239 & Pu-240 & Pu-241 & Pu-242 \\
\hline 17.12 & 0.06132 & 0.2256 & 103.3 & 0.003925 \\
\hline
\end{tabular}

For Tank 4,

$$
\begin{aligned}
P u(\text { Tank } 4)= & 5.997 E 2 / 0.06132+1.336 E 2 / 0.2256+5.365 E 2 / 103.3 \\
& +2.749 E-2 / 0.003925 \\
= & 10,384 g
\end{aligned}
$$

The total Pu in Tanks $7(30 \%), 12$ and 14 are calculated similarly, and the total Pu in Macro-batch 4 is then:

$$
\begin{aligned}
P u(M B 4) & =10,384+19,128+51,089+1,084 \\
& =81,685 g
\end{aligned}
$$

So, the mass-averaged weight percent of $\mathrm{Pu}-238$ then becomes:

$$
\begin{aligned}
w t \% P_{u}-238 & =\frac{1.203 E 5+2.001 E 2}{(17.12)(81,685)}(100) \\
& =8.62
\end{aligned}
$$

Similarly,

$$
\begin{aligned}
w t \% P u-239 & =\frac{5.977 E 2+(3.666 E 3)(0.3)+2.316 E 3+6.045 E 1}{(0.06132)(81,685)}(100) \\
& =81.33
\end{aligned}
$$


Calculation of Decay Heat by Pm-147, Am-241 and Cm-244

The following curie inventory data are obtained from the WCSystem database:

\begin{tabular}{|cccccccc|}
\hline Tank & $\begin{array}{c}\text { Pm-147 } \\
(\mathrm{Ci})\end{array}$ & $\begin{array}{c}\text { Pu-238 } \\
(\mathrm{Ci})\end{array}$ & $\begin{array}{c}\mathrm{Pu}-239 \\
(\mathrm{Ci})\end{array}$ & $\begin{array}{c}\text { Ingrown } \\
\text { Am-241 }\end{array}$ & $\begin{array}{c}\mathrm{Am}-241 \\
(\mathrm{Ci})\end{array}$ & $\begin{array}{c}\mathrm{Cm}-244 \\
(\mathrm{Ci})\end{array}$ & $\begin{array}{c}\text { Tank Total } \\
(\mathrm{Ci})\end{array}$ \\
\hline 4 & $1.893 \mathrm{E}+05$ & & $5.977 \mathrm{E}+02$ & $1.291 \mathrm{E}+03$ & $1.128 \mathrm{E}+04$ & $3.864 \mathrm{E}+00$ & $9.751 \mathrm{E}+06$ \\
7 & $5.727 \mathrm{E}+03$ & & $3.666 \mathrm{E}+03$ & $7.924 \mathrm{E}+03$ & $1.280 \mathrm{E}+04$ & $3.018 \mathrm{E}+00$ & $8.811 \mathrm{E}+06$ \\
12 & $4.073 \mathrm{E}+04$ & $1.203 \mathrm{E}+05$ & $2.316 \mathrm{E}+03$ & $3.735 \mathrm{E}+04$ & $1.347 \mathrm{E}+04$ & $8.055 \mathrm{E}+01$ & $2.023 \mathrm{E}+07$ \\
14 & $1.557 \mathrm{E}+02$ & $2.001 \mathrm{E}+02$ & $6.045 \mathrm{E}+01$ & $1.294 \mathrm{E}+02$ & $6.328 \mathrm{E}+02$ & $5.582 \mathrm{E}-01$ & $4.725 \mathrm{E}+05$ \\
\hline
\end{tabular}

In addition, the following specific curie data in $\mathrm{Ci} / \mathrm{gm}$ are used: ${ }^{11}$

\begin{tabular}{lll}
\hline Pm-147 & Am-241 & Cm-244 \\
\hline 927.6 & 3.416 & 80.88 \\
\hline
\end{tabular}

The total amount of Pu-239 in Macro-batch 4 is:

$$
\begin{aligned}
\text { Pu } 239 & =(81,685)(81.33) / 100 \\
& =66,434 \mathrm{~g}
\end{aligned}
$$

The mass ratio of $\mathrm{Pm}-147$ to $\mathrm{Pu}-239$ is then:

$$
\begin{aligned}
M R(P m / P u) & =[1.893 E 5+(5.727 E 3)(0.3)+4.073 E 4+1.557 E 2] / 927.6 / 66,434 \\
& =0.003763
\end{aligned}
$$

Similarly, the mass ratios of Am-241 and Cm-244 to Pu-239 are:

$$
\begin{aligned}
M R(A m / P u) & =\left[\begin{array}{l}
1.291 E 3+1.128 E 4+(7.924 E 3+1.280 E 4)(0.3) \\
+3.735 E 4+1.347 E 4+1.294 E 2+6.328 E 2
\end{array}\right] / 3.416 / 66,434 \\
& =0.31 \\
M R(C m / P u) & =[3.864+(3.018)(0.3)+(80.05)+0.5582] / 80.88 / 66,434 \\
& =1.6 E-5
\end{aligned}
$$

The material balance showed that the sludge fraction of total $\mathrm{Pu}$ in glass is $8.593 \mathrm{~g} / \mathrm{hr}$ per $228 \mathrm{lb} / \mathrm{hr}$ glass production. ${ }^{3}$ So, the total Pu-239 in one Macro-batch 4 canister is:

$$
\begin{aligned}
M(P u-239) & =(8.593)(0.8133)(4,000 / 228) \\
& =122.609 \mathrm{~g}
\end{aligned}
$$


So, the mass of Pm-147 in one Macro-batch 4 canister is:

$$
\begin{aligned}
M(P m-147) & =(122.609)(0.003763) \\
& =0.4614 g
\end{aligned}
$$

The decay heat generated by $\mathrm{Pm}-147$ is then:

$$
\begin{aligned}
D H(P m-147) & =(0.4614)(0.34) \\
& =0.157 \mathrm{Watt}
\end{aligned}
$$

Similarly,

$$
\begin{aligned}
D H(A m-241) & =(122.609)(0.31)(0.113) \\
& =4.295 \text { Watt } \\
D H(A m-241) & =(122.609)(1.6 E-5)(2.78) \\
& =0.0055 \text { Watt }
\end{aligned}
$$


WSRC-TR-98-00466

CALCULATION OF DWPF CANISTER DECAY HEAT FOR SLUDGE MACRO-BATCHES $1 B$ TO 9 (U)

Distribution:

W. D. Kerley, 704-S

J. F. Ortaldo, 704-S

R. E. Edwards, 704-25S

J. A. Gentilucci, 704-18S

L. M. Papouchdo, 773-A

E. W. Holtzscheiter, 773-A

L. F. Landon, 704-1T

S. M. Marra, 704-1T

M. J. Mahoney, 703-H

N. R. Davis, 703-H 